\title{
Wie technische Systeme aus uns schlau werden. Von maschineller Auslegung und Festlegung
}

\section{Bruno Gransche}

\section{Zusammenfassung}

Das Zusammenwirken von natürlicher und künstlicher Intelligenz kann aus philosophischer Perspektive als ein Fall von allgemeineren Mensch-TechnikRelationen gefasst werden. Dieser Beitrag nimmt einerseits eine solche allgemeinere Perspektive ein, betrachtet andererseits die Assistenzrelation zwischen lernenden Assistenzsystemen und Menschen als wiederum einen Fall solchen Zusammenwirkens.

Dazu werden zunächst einige Begriffe abgeschritten, die die philosophische Perspektive etwa im Gegensatz zur informatischen oder psychologischen benötigt. Nach einigen handlungstheoretischen Grundlagen rückt die Perspektive auf aktuelle und im Entstehen befindliche Mensch-Technik-Relationen in den Mittelpunkt. Als ein Unterfall von Mensch-Technik-Welt-Verhältnissen hat Don Ihde die sogenannte hermeneutische Relation herausgestellt. Diese besagt: Menschen interpretieren die Welt vermittelt durch Technik. Kern des hier vorliegenden Beitrags ist die These von der Inversion dieser hermeneutischen Relation durch lernende Technik bzw. künstliche Intelligenz. Diese Inversion besagt: Technik, interpretiert' Menschen vermittelt durch Daten und arrangiert entsprechend die Welt. In beiden Relation geht es um deuten und gedeutet werden und damit immer auch um den Kampf um Deutungshoheiten.

B. Gransche $(\square)$

Institute of Advanced Studies FoKoS, University of Siegen, Siegen, Deutschland E-Mail: mail@brunogransche.de

(C) The Author(s) 2021 


\section{Schlüsselwörter}

Technikphilosophie · Digitalisierung · Maschinelles Lernen ·

Technikhermeneutik · Technische Assistenzsysteme · Postphänomenologie ·

List der Systeme

\section{Begriffliches zur interdisziplinären Verständlichkeit}

Statt von Natürlicher Intelligenz. (NI) und Künstlicher Intelligenz (KI) soll hier von Mensch und Technik die Rede sein. Grund dafür sind einerseits gewisse Nachteile der Begriffe NI und KI und andererseits Vorteile der Begriffe Mensch und Technik. Es ist nämlich weder in der öffentlichen Debatte noch den verschiedenen Disziplinen inklusive der Philosophie klar, was natürlich, künstlich oder Intelligenz denn überhaupt sein sollten. Der Begriff der Intelligenz teilt seine Unbestimmtheit mit weiteren unerlässlichen, aber notorisch vieldeutigen Begriffen wie Information, Leben (z. B. bios oder zoe), Kultur, aber auch Mensch und Technik. Mit der hier vorgenommenen Begriffsdifferenzierung soll vor allem Aufmerksamkeit darauf gelenkt werden, dass Begriffe wie KI oder auch Autonome Technik, die derzeit große politische, wissenschaftliche und wirtschaftliche Aufmerksamkeit erfahren ${ }^{1}$, vielfältige und teils widersprüchliche Vorstellungen oder Phänomene in sich vereinen. Im Gegensatz zur Intelligenz werden die Begriffe Mensch und Technik in der Philosophie einerseits als Inbegriffe (Menge von kategorial verschiedenen Entitäten, die von einem gemeinsamen Interesse zusammengehalten werden ${ }^{2}$ ) reflektiert (etwa Technik als Inbegriff der Mittel). ${ }^{3}$ Andererseits sind sie philosophisch präsent als Reflexionsbegriffe, also Namen

\footnotetext{
${ }^{1}$ Etwa die KI-Strategie der Bundesregierung von 2018 (Bundesregierung 2018) oder die Einigung von 42 Ländern auf einen einheitlichen Umgang mit KI-Entwicklungen unter Empfehlung des Rats zu künstlicher Intelligenz der OECD von 2019 (OECD 2019).

${ }^{2}$,Ein Inbegriff entsteht, indem ein einheitliches Interesse und in und mit ihm zugleich ein einheitliches Bemerken verschiedene Inhalte für sich heraushebt und umfasst. [...] Fragen wir, worin die Verbindung bestehe, wenn wir z. B. eine Mehrheit so disparater Dinge wie die Röte, der Mond und Napoleon denken, so erhalten wir die Antwort, sie bestehe bloss darin, dass wir diese Inhalte zusammen denken, in einem Acte denken." (Husserl 1891, S. 79).

${ }^{3}$ Dies wäre eine Technik-Definition, die hier nicht geteilt wird, aber als Beispiel taugt. Vgl. zur Kritik an Technik als Inbegriff der Mittel (Hubig 2006).
} 
von Strategien, unter denen Vorstellungen über Technik oder Mensch, Menschliches und Nichtmenschliches oder Technisches und Nichttechnisches erzeugt werden. ${ }^{4}$ Schließlich hat Intelligenz gewissermaßen einen a-philosophischen Klang, da in der Philosophie eher Weisheit, Klugheit oder Besonnenheit diskutiert wurden. Mit dem Adjektiv schlau versucht dieser Beitrag bereits im Titel begrifflich eine philosophische Sicht auf Intelligenz anzukündigen. Wenn dabei wie von NI und KI als Kontrastbegriffen die Rede ist, lohnt es nicht, in Scheinantagonismen und Abgrenzungsdispute zu verfallen, wie es die Anthropologie etwa lange zur Frage Was ist der Mensch? getan hat, da jegliche Grenzziehung die Grenzübertretung schon heraufbeschwört. Ironisch konterkariert wurde diese Dynamik bereits in der Antike durch den Hahn des Diogenes. ${ }^{5}$ Fruchtbarer wäre es also, statt von Mensch, Technik, Natur oder dem Künstlichen zu sprechen, erkenntnisgeleitet $\mathrm{zu}$ hinterfragen, welche Aspekte an einem komplexen Phänomen - bspw. Intelligenz, Klugheit, Lernfähigkeit - etwas Menschliches, Technisches, Natürliches oder Künstliches aufweisen und was daraus etwa an Übertragbarkeit folgt.

Bei der Annäherung über die Begriffe Mensch und Technik muss wie bei NI und KI beachtet werden, dass dabei zwar oft der Singular gebraucht wird Kollektivsingulare sind mitunter ein Indiz für Vorurteile -, dass aber eine wesentliche und oft unterschlagene Frage diejenige ist, welche Menschen oder Systeme jeweils gemeint sind. Ob etwa die Technik bald den Menschen intelligenzleistungsmäßig übertreffe, hängt stark davon ab, welche Technik (unsere alltäglichen consumer electronics, Forschungsprototypen oder Höchstleistungsrechenzentren?) welche Menschen (welchen Bildungsstandes, mit welchen materiellen, geistigen und sozialen Ressourcen?) worin genau (Schachspielen, Schachboxen oder Schachturnier-Regeln erfinden?) übertrifft.

In diesem Beitrag ist zudem von Assistenzsystemen die Rede, womit im weitesten Sinne lernende Systeme gemeint sind, die bereits heute oder in naher Zukunft im Alltag der Menschen mitwirken; Systeme, die über maschinelles Lernen datenintensiv ihr Systemverhalten personalisiert situationsspezifisch adaptieren. Assistenz durch solche Systeme wird verstanden als Sonderfall von Mensch-Technik-Relationen, welche wiederum nur einen Fall neben den

\footnotetext{
${ }^{4}$ Vgl. zu Technik, Natur und Kultur als Inbegriffe und als Reflexionsbegriffe (Hubig 2011). ${ }^{5}$ „Als Platon definierte: ,Ein Mensch ist ein zweifüßiges Tier, das ungefiedert ist", und Billigung fand, rupfte Diogenes einen Hahn, brachte ihn in die Schule mit und sagte: ,Das ist der Mensch des Platon'. Daher wurde dem Begriffe das Merkmal ,breitnagelig“ hinzugesetzt.“ (Kirchner et al. 1998, S. 254).
} 
technisch vermittelten Mensch-Mensch-Relationen darstellen. Im Assistenzverhältnis wird in besonderer Weise ein Zusammenwirken von menschlichen/ natürlichen und technischen Leistungsmerkmalen in den Blick genommen. Des Weiteren wird Mensch-Technik-Zusammenwirken als eine Art des Agierens verstanden, das als Hybrid aus menschlichen Handlungsaspekten und technischem Prozessieren zu verorten wäre zwischen Handlungen (von Menschen intendiert herbeigeführten Ereignissen) und Vorgängen (von Menschen nicht herbeigeführten Ereignissen). ${ }^{6}$ Eine solche Aktion würde sich als Interaktion darstellen, wenn die Aktionen der einen Instanz auf die (Re-)Aktionen der anderen Instanz gerichtet sind und sich Erwartungserwartungen über das Wechselspiel bilden. Sie würde sich als Koaktion darstellen, wenn zahlreiche menschliche und technische Aktionsinstanzen parallel zueinander zu einem Gesamtergebnis ursächlich beitragen, ohne ihre Einzelaktionen aufeinander und gemeinsam auf das Ziel zu richten (wie etwa im Börsenhandel) (Vgl. zu Aktion, Interaktion und Koaktion Gransche et al. 2014).

\section{Assistenzimpertinenz und Systemlist}

„FAUST: Mein schönes Fräulein, darf ich wagen, Meinen Arm und Geleit Ihr anzutragen? MARGARETE: Bin weder Fräulein, weder schön, Kann ungeleitet nach Hause gehn." (Goethe 1986, S. 75). Diese Szene verdeutlicht eine Ambivalenz von Hilfe, die in den Schmieden der technischen Unterstützungssysteme oft naiv auf ihre positive Seite verengt wird; nämlich die eigentlich ungerechtfertigte Annahme, dass Menschen Hilfe wollen. Im Gegensatz zu Smart Everything (Smart home etc.) Assistenzsystemen fragt Faust hier immerhin noch, ob mit dem Arm unterstuitzt und begleitet bzw. geleitet werden darf eine Wahl vor die die meisten Systemnutzer heute kaum mehr gestellt werden. ${ }^{7}$

\footnotetext{
${ }^{6}$ Neben nicht herbeigeführten Ereignissen (Vorgängen) und intendiert herbeigeführten Ereignissen (Handlungen) wären noch die nicht intendiert herbeigeführten Ereignisse (Verhalten) abzugrenzen (vgl. Gransche und Gethmann 2018).

${ }^{7}$ Es ließe sich einwenden, dass man eine entsprechende App ja nicht installieren oder ein entsprechendes System nicht nutzen müsse; über mehrheitliche Nutzung entstehen aber soziale Zwänge sowie massive Gratifikationsentzüge durch strafende Netzwerkeffekte, die man sich leisten und die man aushalten können muss. So muss im strengen Sinne heute niemand eine Emailadresse oder ein Bankkonto haben, allerdings um den Preis, an weiten Teilen der Gesellschaft (z. B. den meisten Arbeitsplätzen) nicht mehr teilnehmen zu können. So wird eine prinzipielle Wahl faktisch zum praktischen Zwang.
} 
Arm und Geleit kommen selten als harmloses Hilfsangebot daher und Faust hofft hier auf intime Gegenleistung durch Gretchen. Diese durchschaut das und weist das Gesamtpaket aus Hilfsangebot und Erwiderungserwartung als Assistenzimpertinenz mit dem Hinweis Ich kann selbst! von sich: „Kann ungeleitet nach Hause gehn“ (Goethe 1986, S. 75) - wer kann das gegenüber Google Maps und Navigationssystemen unserer Autos heute noch von sich behaupten? ${ }^{8}$ Es werden wohl immer weniger. ${ }^{9}$ Mit intimen Gegenleistungen wird Assistenzsystem-Geleit aber auch in diesen Fällen bezahlt - wenn noch nicht mit Beischlaf, so doch mit intimsten Einblicken und Auskünften (Daten). Nur durchschauen wir das weniger, als Gretchen Faust durchschaut. ${ }^{10}$ Würden wir nicht sonst öfter die Assistenzimpertinenz des Smart Everything mit dem Hinweis ablehnen: Ich kann selbst!? Wir begreifen dies weniger als jeder, der weiß, dass die Frage Can I buy you a drink? selten Trinken zum Gegenstand hat - genauer: Die Frage präsentiert Trinken zwar als Zweck, verbirgt jedoch einen anderen Zweck, zu dem Trinken wiederum das Mittel ist. Analog präsentiert bspw. Spotify mit seinem Streaming Angebot ein Mittel zum Zweck des Musikkonsums, verbirgt aber einen dahinterstehenden Zweck der Nutzerprofilierung, zu dem Musikkonsum selbst nicht mehr Zweck, sondern wiederum Mittel ist. Das Vorzeigen eines vermeintlichen bei gleichzeitigem Verbergen eines eigentlichen Zwecks, bzw. die Transformation eines Zwecks (Assistenz, Drinks, Musik) in ein Mittel zu einem anderen Zweck

\footnotetext{
${ }^{8}$ Aus technisch informierter Sicht wäre das Phänomen der Assistenzimpertinenz noch verstärkt aufseiten adaptiver Automation zu finden - bei dieser geht die Optionenselektion und Interaktionsaufteilung zwischen Mensch und System vom System aus, der Mensch bekommt Optionen nach Maßgabe des Systems zugeteilt. Im Gegensatz dazu steht die adaptierbare Automation, bei der der Mensch entscheidet, wie die Optionen und Interaktionsanteile zugeteilt werden (Kaber und Prinzel 2006). Eine ähnliche Anpassungsdifferenz findet sich bei der Personalisierung (vgl. Peppers und Rogers 1997), bei der systemseitig Anpassungen vorgenommen werden, und der Individualisierung (vgl. Davis 1997), bei der die Anpassungen von den Menschen bzw. Nutzern ausgehen.

${ }^{9}$ Auch hier hat man zwar prinzipiell noch die Wahl, z. B. sich ohne Navigationsdienste zu orientieren. Hier zeigt sich neben den praktischen Zwängen, aber eine weitere Dimension: in dem Maße, in dem Assistenz angenommen wird, schwindet die Kompetenz bezüglich der dauerhaft delegierten Aufgabe. Wer sich also dauerhaft nach Hause geleiten lässt, kann irgendwann nicht mehr ungeleitet nach Hause gehen (vgl. Münzer et al. 2012).

${ }^{10}$ Hier gibt es sicher prinzipielle Durchschauens-Unterschiede; informierte, mündige Nutzer wären dazu eher in der Lage als weniger informierte. Nur steigt die Anforderung, adäquat informiert zu sein und zu bleiben - zeitlich wie bezüglich Bildung und kognitiver Kapazitäten - mit jedem zusätzlichen System, jeder zusätzlichen Systemkomponente und Drittanbieterdienst sowie mit steigender Vernetzung und Komplexität der Systeme selbst.
} 
(physischer oder informationeller Intimkontakt) ist listig; verborgene Zwecktransformation ist die Struktur der List. ${ }^{11}$

Im Falle von Arm und Geleit, den Free Drinks oder dem schon fast sprichwörtlichen Kaffee, auf den man noch mit reinkommt, ist die List offengelegt. ${ }^{12}$ Es gibt hier also keine wirklich Überlisteten, sondern allenfalls Mitspieler, die sich wiederum listig als Überlistete darstellen. Solcherlei Listen werden durch entsprechende Kulturtechniken - Rituale, Scripts, Üblichkeiten - schadlos gehalten; sie können - analog dazu, was Robert Pfaller aufbauend auf Kants ,schuldlose Täuschung“ (Kant 1798, S. 151) „Lügen ohne Belogene“ oder „weiße Lügen“ (Pfaller 2018, S. 70-111) nennt - als weiße Listen bezeichnet werden. Kaum jemand, der mit dem entsprechenden Mitspieler die gleichen Kulturtechniken teilt, würde sich im genannten Beispiel bei Beginn der Intimitäten empört um den Kaffee betrogen sehen. Aus der Rolle, Mitspieler zu sein, resultieren keine Anspruchsrechte der anderen Mitspieler und keine Pflicht weiter zu spielen, wohl aber strukturiert die Zugehörigkeit zum Spiel (Irrtümer darüber, wo man gerade mitspielt, sind möglich!) die Erwartung und Angemessenheitsbewertungen der Beteiligten.

\footnotetext{
${ }^{11}$ Dieses Schema zeigt auch Hegels „List der Vernunft“ und eine solche Überlistung charakterisiert er als Gewalt: „Daß der Zweck sich unmittelbar auf ein Objekt bezieht und dasselbe zum Mittel macht, wie auch daß er durch dieses ein anderes bestimmt, kann als Gewalt betrachtet werden [...]. Daß der Zweck sich aber in die mittelbare Beziehung mit dem Objekt setzt und zwischen sich und dasselbe ein anderes Objekt einschiebt, kann als die List der Vernunft angesehen werden.“ (Hegel 1999, S. 452).

${ }^{12}$ Für welche Nutzer Angebote wie das von Spotify quasi Einblicke in die tatsächlichen Tauschgehalte nicht (mehr) als List, sondern als bewusster Deal erscheint, wäre wiederum relativ zum Wissensstand der Nutzer. Auf unbestimmte Art hat sich der Daten-gegenService-Deal herumgesprochen. Genauso aber haben sich in antiken Kriegen schon trickreiche Invasionstaktiken herumgesprochen. Das schützte Troja nicht vor dem Untergang durch die List des Trojanischen Pferdes. Das tatsächliche Ausmaß des Datendeals schockiert vermeintlich mündige Nutzer wie Profis. Dieser Schock ist der Moment, in dem die eigene Überlistung bewusst wird; beispielsweise eine The Guardian Journalistin, die bei dem Datingservice Tinder Auskunft über ihre Daten verlangte: ,Some 800 pages came back containing information such as my Facebook, likes', links to where my Instagram photos would have been had I not previously deleted the associated account, my education, the age-rank of men I was interested in, how many Facebook friends I had, when and where every online conversation with every single one of my matches happened ... the list goes on." (Duportail 2017). Das Datenausmaß kann selbst Datenwissenschaftler erschrecken: „I am horrified but absolutely not surprised by this amount of data, " said Olivier Keyes, a data scientist at the University of Washington." Ebd.
} 
Aus technikphilosophischer Sicht ist grundsätzlich zu konstatieren, dass jeder, der Technik nutzt, listig ist. Technik ist die List der Naturüberwindung und die sprachliche griechische Wurzel der Mechanik - mechane - bedeutet wörtlich List (daher auch der Beiname des Odysseus polymechanos, der Listenreiche). Technik taugt aber nicht nur zur Naturüberlistung, sondern auch zur Überlistung anderer Menschen sowie zur Selbstüberlistung. ${ }^{13}$ Im Falle der Listen der Systeme ${ }^{14}$ handelt es sich derzeit meist gerade nicht um weiße Listen, und dies so lange nicht, wie die entsprechenden Kulturtechniken die Verbergensstrategien der Listigen nicht selbstverständlich transparent machen. Im Falle entsprechender datengetriebener Geschäftsmodelle wie der von Google, Facebook, Amazon, Apple oder - wenn auch erheblich kleiner - Spotify gibt es zweifellos Überlistete, Getäuschte und Belogene. Geschäftsmodelle beruhen gerade darauf, dass die Nutzer ihr Nutzungsverhalten auf den vermeintlichen und eben nicht auf den verborgenen Zweck richten. Wer Amazon Alexa nutzt, tut dies unter der - irrigen - Annahme, dass es sich bei dem Person-Alexa-Verhältnis lediglich um ein Assistenz- oder Dienstleistungsverhältnis von Alexa gegenüber der Person handelt, man also für sein Echo zahlt und im Gegenzug den Wasserkocher aktiviert oder Rezepte vorgelesen bekommt. Wer Spotify Premium (die kostenpflichtige und dafür werbefreie Variante) nutzt, wähnt sich in einem Musikkonsumverhältnis ähnlich dem Plattenkauf - nur, dass nicht etwa jeden Monat eine Platte gekauft und gehört würde, sondern ein Monatsbeitrag zum Zugriff auf Millionen Platten berechtigt. Während aus Sicht des Nutzers der Musikkonsum nach diesem anachronistischen Verständnis durchaus Zweck des Geschäftsverhältnisses zwischen Individuum und Spotify sein mag, so ist er für Spotify - dann als Spotify for Brands - Mittel zum Zweck der Profilbildung. Auf Spotify (2018) heißt es dazu:

Je mehr unsere Nutzer streamen, desto mehr erfahren wir über sie. Das Engagement unserer Nutzer ist der Treibstoff für unsere Streaming Intelligence. Unsere Insights zeigen, wer die Menschen hinter den Geräten wirklich sind. Diese personenbezogenen Echtzeit-Einblicke gehen weit über demografische Angaben und Geräte-IDs hinaus und zeigen auch die Stimmungen, Einstellungen, Vorlieben und Verhaltensweisen unserer Hörer. Wir haben festgestellt, dass das StreamingVerhalten viel über den einzelnen Hörer aussagt. [...] Und das Beste: Die neuen Forschungsergebnisse lassen immer öfter auch Rückschlüsse auf das Offline-Verhalten der Streaming-Generation zu.

\footnotetext{
${ }^{13}$ Mythologische Ikone einer solchen Selbstüberlistung ist wiederum Odysseus, als er sich zum Schutz vor seiner eigenen Verführbarkeit angesichts der Sirenen an den Mast binden lässt.

${ }^{14}$ Vgl. zum Gedanken der List der Systeme auch: (Gransche 2020).
} 
Das Engagement der Nutzer erscheint zwar als erkauftes Nutzungsrecht für Abonnenten; für Spotify ist es aber nicht nur ein Mittel, sondern ein Verbrauchsgut, nämlich Treibstoff für lernende Systeme, die Streaming Intelligence. Spotify könnte diese List transparent machen und statt mit „Spotify Premium macht glücklich.“ (Spotify Werbeslogan von 2019; spotify 2019) etwa werben mit: Verraten Sie uns mit ihrem Musikkonsum alles über ihren Alltag, ihre Tätigkeiten, Stimmungen, Charaktermerkmale und Konsumpräferenzen für nur 9,99€ im Monat. Das Geschäftsmodell von Spotify stünde zumindest in Frage, wenn es seine List so als weiße kommunizieren würde. Millionen Nutzer nutzen täglich Spotify und es ist äußerst fraglich, wie viele von diesen hinter dem vermeintlichen Zweck des Musikkonsums von Spotify auch den verborgenen Zweck von Spotify for Brands kennen und bewusst in Kauf nehmen, mit der Konsequenz:

We know our fans. Like, really know them. That time you skipped Britney to hear The Beatles. That time you played 'Young Dumb \& Broke' 117 times in a row. That time you made a Road Trip playlist with your friends. Every swipe, search, skip, and shuffle tells us a story about our audience. (spotify for brands, Abgerufen am 03.12.2018)

Was tut Spotify - und was tun in weit größerem Ausmaß die Branchenriesen Google, Amazon, Apple, Facebook etc. - mit diesem Wissen? Die beiden Grafiken zeigen einerseits, wie viele Internetseiten (Abb. 1) und andererseits, wie viele Unternehmen (Abb. 2) schon während einer bloß 60-min Streaming-Session involviert sind. ${ }^{15}$

Um in obiger Analogie zu bleiben, kann man davon ausgehen, dass Millionen von Nutzern bei lernenden Systemen (und deren Betreibern) auf einen Kaffee mit hereinkommen und naiverweise tatsächlich glauben, nur Kaffee zu bekommen. Allerdings - und hier zeigt sich ein weiterer Unterschied - findet mit Systemen der anschließende Intimkontakt für den Datentreibstoff Individuum nicht direkt wahrnehmbar statt, sondern wirkt über Folgeoptionen, beispielsweise für Kredite, erst über den Umweg der beteiligten Unternehmen, die Kunden der Intimkenntnis-Hehler sind. Wo Listen genutzt werden und es sich nicht um weiße Listen handelt, stellt sich normativ die Frage, welche Dimensionen des Verbergens als unzulässig zu gelten hätten. Da es sich um Geschäftsmodelle handelt,

\footnotetext{
${ }^{15}$ Die Bilder zeigen in dieser Auflösung lediglich den hier fokussierten Punkt, dass es sich um eine sehr große Anzahl handelt. Die einzelnen Knotenbeschriftungen in den Grafiken lassen sich nachvollziehen in der Originalquelle (Mähler und Vonderau 2017).
} 


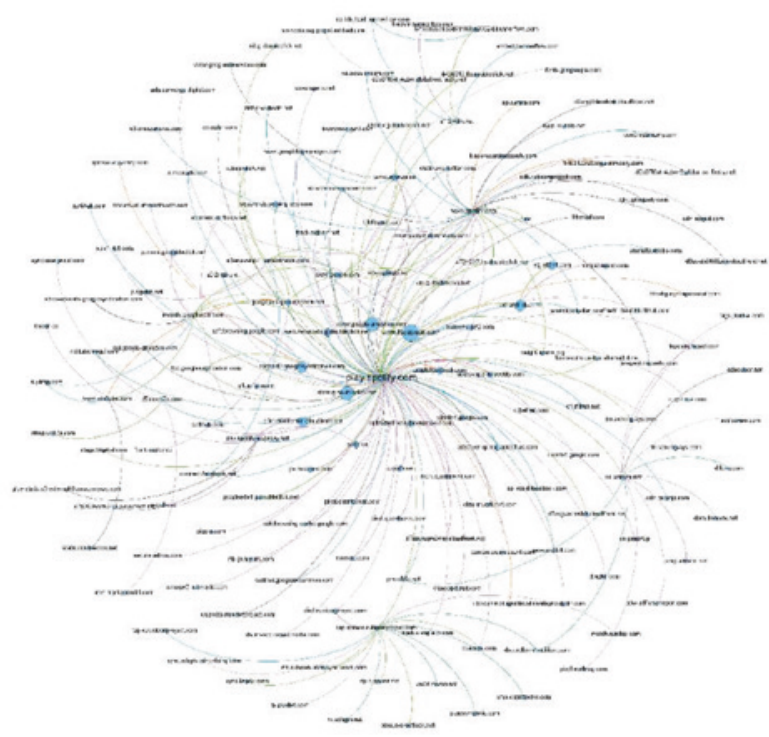

Abb. 1 Quelle: Mähler und Vonderau (2017, S. 218)

liegt als ein Beispiel solcher Normen eine wettbewerbsrechtliche Norm nahe. $\S 5$ des Gesetzes gegen den unlauteren Wettbewerb (UWG) besagt: „Unlauter handelt, wer eine irreführende geschäftliche Handlung vornimmt, die geeignet ist, den Verbraucher oder sonstigen Marktteilnehmer zu einer geschäftlichen Entscheidung zu veranlassen, die er andernfalls nicht getroffen hätte." (Bundesamt für Justiz 2004b). Jemanden zu einer Entscheidung zu veranlassen, die er andernfalls nicht getroffen hätte, ist häufig Ziel der List. Die Urlist des listenreichen Odysseus, das trojanische Pferd, veranlasste die Troer, ihre Tore zu öffnen. Ohne Odysseus' List hätten sie sich schließlich anders entschieden und ihre Tore geschlossen gehalten. Der Großteil der Intentionen derer, die Datenprofile von Spotify, Google und Co. kaufen, richtet sich auf die Entscheidungs- und Handlungsbeeinflussung, was unter dem Begriff Nudging 2017 mit einem sogenannten Wirtschaftsnobelpreis prämiert wurde. Konstitutives Element einer jeden List sind Verbergensstrategien, die diejenigen, vor denen sie etwas verbergen, entsprechend in die Irre führen. „Unlauter handelt, wer im konkreten Fall unter Berücksichtigung aller Umstände dem Verbraucher eine wesentliche Information vorenthält.“ (Bundesamt für Justiz 2004a). Explizit Datentreibstoff und nicht nur 


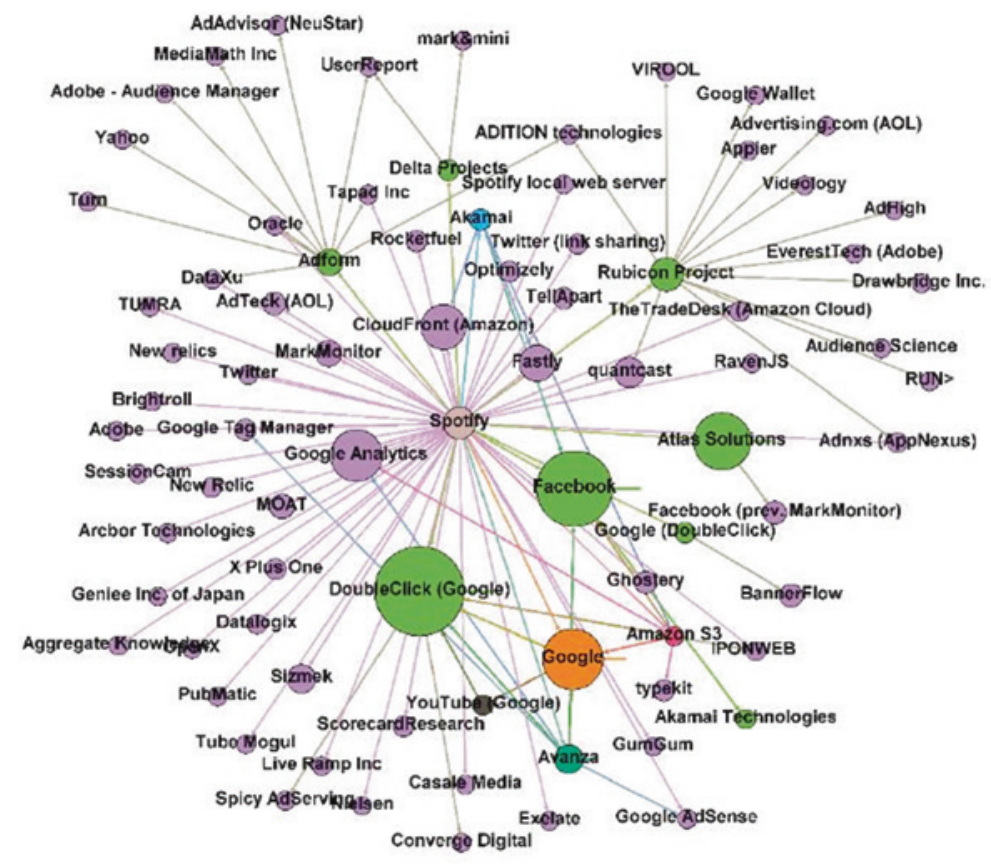

Abb. 2 Quelle: Mähler und Vonderau (2017, S. 219)

Musikkonsument zu sein, ist, zumindest für den rechtlichen Laien, eine wesentliche Information. Dies stellt die systemisch listige Entscheidungs- und Handlungsbeeinflussung damit zumindest - abgrenzend zur weißen List - unter Unlauterkeitsverdacht.

\section{$3 \quad$ Zwecksetzungsassistenz - Hilf mir zu wollen!}

Wer mehr erreichen will, als er alleine erreichen kann, muss sich entweder Hilfe organisieren, oder zu einer List der Widerstandsüberwindung, nämlich Technik greifen. In einer Assistenzrelation als Unterfall der Mensch-Mensch-Relationen kann der Assistierte Handlungszwecke realisieren, zu deren Erreichung seine eigenen Mittel und Vermögen nicht genügen. In der Assistenzrelation werden die Kompetenzen des Assistierten um die Kompetenzen des Assistenten so erweitert, dass ein Handlungsziel entweder überhaupt oder sehr viel müheloser erreicht 
werden kann. Zur Motivation des Assistenten muss entweder eine Einigung über die gemeinsame Zwecksetzung herbeigeführt werden - und sei es die Einigung auf Inkaufnahme eines Aufwandes zur Erreichung einer Vergütung -, oder aber, die unterstützungsleistende Seite muss zur Leistungserbringung gezwungen werden. In zwischenmenschlichen Assistenzrelationen werden also Leistungsvermögen entweder durch Einigung oder durch Unterdrückung zusammengeführt. Alleine kann ein Handelnder seine Zwecksetzungsautonomie ungehindert nutzen, ist aber in der Dimension infragekommender Zwecke durch sein Leistungsvermögen begrenzt. Durch die Kooperation erweitern sich die Zweckoptionen, jedoch müssen in der Einigung Kompromisse eingegangen, also vom eigenen Willen ein Stück abgerückt werden. Unterdrückung andererseits erfordert Macht und ruft Gegenkräfte hervor. Der Schlaraffenland-Traum anstrengungsloser Unmittelbarkeit der Zweckerfüllung mittels technischer Systeme stellt den Versuch dar, ohne Einigung und ohne Unterdrückung, also bei voller Zwecksetzungsautonomie dennoch mehr, andere und größere Zwecke realisieren zu können. Wem Alexa auf Zuruf das Licht einschaltet, der muss weder selbst aufstehen, noch sich mit den anderen Anwesenden einigen, noch einen Hausbediensteten für das Einschalten bezahlen oder einen Haussklaven dazu zwingen. Mit der zunehmenden Autonomie - eigentlich zunehmend höherstufiger Automatisierung ${ }^{16}$ - der Systeme werden immer mehr Aufgaben delegierbar. Damit wächst die Menge der Zwecke technisch-assistierten Handelns. Gleichzeitig verändern sich die menschlichen Kompetenzen im Maße ihrer tatsächlichen Delegation - use it or lose it. Der assistierte Mensch kann immer mehr erreichen, aber immer weniger davon noch selbst. Mit einem Navigationssystem findet man überallhin, auch zu Destinationen, deren Erreichen zuvor aus Unkenntnis nicht als realisierbare Möglichkeit zur Verfügung stand. Wer aber täglich nur noch dem Navigationssystem folgt, der findet bald ohne es nicht einmal mehr eigentlich

\footnotetext{
${ }^{16}$ Die Rede von autonomer Technik ist - ähnlich der von KI - aus philosophischer Sicht fragwürdig, aber derart verbreitet, dass sie sich durchgesetzt hat. Auf technischer Seite stehen verschiedenste - im Wandel begriffene; bspw. in der kognitiven Robotik (Schilling et al. 2016) - Konzepte der Autonomie, wovon keine mit der philosophischen Konzeption der Autonomie bspw. bei Kant in eins fällt (vgl. Gransche et al. 2014; Gottschalk-Mazouz 2008). Genauer wäre im technischen Bereich von Automation und Automatisierungsgraden zu sprechen, z. B. die fünf Automatisierungsstufen von acatech (acatech 2015, S. 11), wobei hier in problematischer Weise häufig die fünfte Stufe wiederum als ,vollständige Autonomie" bezeichnet wird (Hightech Forum und acatech 2017, S. 91-92). Die Level of Automation (LOA) haben Vorläufer bspw. bei (Sheridan und Verplank 1978; Endsely und Kaber 1999).
} 
bekannte Ziele (vgl. Münzer et al. 2012). Unabhängig von künftiger Leistungsfähigkeit von Assistenzsystemen gilt erstens, dass Kompetenzaufbau und -erhalt nie delegierbar sein werden: Wer einen virtuosen Roboter $24 \mathrm{~h}$ am Tag für sich Klavier üben lässt, lernt nicht selbst spielen. Zweitens ist die normative Entscheidung im Rahmen der Zwecksetzungsautonomie nicht delegierbar, solange die Systeme nicht über ein Eigeninteresse, ein postbiotisches Bewusstsein oder Künstliche Intelligenz im starken Sinne verfügen: Ein autonomes (hochautomatisiertes) Auto kann Drehzahl und Geschwindigkeit, kann Fahrstil und Routenführung (z. B. stauvermeidend nach München zu kommen) autonom wählen, es wird jedoch nie sagen: Ich möchte heute nicht nach München. Auch wenn zwar auf operativer Ebene (Wahl der Mittel) und strategischer Ebene (Wahl der Wege) Aufgaben an die Technik in der Kooperation delegiert werden können: Auf normativer Ebene (Wahl der Zwecke) kann die Leistung nicht an Technik delegiert werden. Im Rahmen der eigenen Zwecksetzungsautonomie zu entscheiden, ist also nicht nur umkämpftes Recht - etwa gegen Unterdrückung und Fremdbestimmung -, sondern auch nicht delegierbare Pflicht autonomer Subjekte. Allerdings sind Zwecke zu setzen und zwischen Optionen zu entscheiden wiederum klarerweise Fähigkeiten, die man lernen und verlernen kann. Während also Assistenzsysteme bislang vor allem bei der Zweckrealisierung, der Handlungsausführung unterstützten, so kommen nun mit den lernenden Systemen vor allem Unterstützungen bei der Zweckentscheidung, der Optionenwahl und Handlungsplanung ins Spiel. Die Zwecksetzung wird nicht delegiert, aber dennoch assistiert. Dies findet sich vor allem in Experten-, Empfehlungs- und Entscheidungsunterstützungssystemen. Diese Form der ,normativen Assistenz bietet Hilfe bei der Qual der Wahl, welche Zahnbürste man kaufen, welche Musik man hören, welche Versicherungen man abschließen, welchen Partner man daten, welche Partei man wählen solle usw. Für all diese Entscheidungen und viele mehr gibt es technische Assistenzen.

Die Technik wird im zweifachen Sinne zuvorkommend. Einerseits verschiebt sich die Assistenzleistung von der Ausführung einer entschiedenen Handlung zeitlich nach vorne manipulativ in die Handlungsplanung und Zweckentscheidung bzw. analysierend in die Handlungsentstehung hinein - wie z. B. bei predictive analytics (Abbott 2014), predictive maintenance (Yan et al. 2017; Cachada et al. 2018), predictive pricing (Wadivkar 2019) etc. So können Systeminterventionen zur Handlungsassistenz proaktiv der eigentlichen Assistenznachfrage zuvorkommen. Andererseits wird die Technik zuvorkommend, insofern sie unter der Maßgabe der Anstrengungsentlastung und der Komfortmaximierung interveniert. Um allerdings schon bei der Zwecksetzung und nicht erst bei der Zweckrealisierung assistieren zu können, müssen Systeme die Nutzer intim 
kennenlernen, bspw. hinsichtlich ihres Streaming- und Konsumverhaltens, ihrer Präferenzen und Alltagsvollzüge. Eine Bedingung für den Vorstoß technischer Assistenzfähigkeit in die Sphäre der Zwecksetzungsautonomie ist daher die Eigenschaft maschinellen Lernens sowie deren Vorbedingungen von entsprechender Rechenleistung, Lernverfahren und Datenmengen.

\section{$4 \quad$ Deuten oder gedeutet werden}

Der Technikphilosoph Don Ihde hatte Ende der 1970er Jahre eine Reihe von Mensch-Technik-Welt-Relationen beschrieben und an Beispielen seiner Zeit verdeutlicht. ${ }^{17}$ In aller Kürze ist Ihdes Position die, dass das Verhältnis von Mensch und Welt durch Technik vermittelt (machine mediated) und also je technikspezifisch transformiert wird. Je nachdem in welchen Technikverhältnissen wir uns dabei befinden, zeigen sich unterschiedliche Effekte der Amplifikation und Reduktion in technisch vermittelter Wahrnehmung gegenüber einer - in Reinform hypothetischen - nackten Wahrnehmung. ${ }^{18}$ Dabei unterscheidet er zunächst die verkörperte Relation (,embodiment relation,“ „experience through machine“; Ihde 1979, S. 6-11), bei der wir Eigenschaften der Welt durch Technik erfahren etwa so, wie ein Zahnarzt mittels einer Sonde kleinste Unebenheiten auf der

\footnotetext{
${ }^{17}$ Er ist seither mit seiner Philosophie auf viele technische Entwicklungen eingegangen. Die erwähnten Relationen aus Technics and Praxis von 1979 sind auch für Nicht-Philosophen sehr eingängig und - vor allem mit Blick auf die Technosphäre und background relations von ungebrochener Aktualität (vgl. Ihde 1979).

${ }^{18}$ Dass es dieser Position nach kein Weltverhältnis gäbe, das nicht irgendwie technisch vermittelt wäre, scheint möglicherweise kontraintuitiv. Dies liegt aber daran, dass dieser Position ein umfassender Technikbegriff und die Vorstellung von Technik als Medium zugrunde liegt: „Während in verkürzter Sichtweise Technik als Inbegriff rational organisierter Handlungsmittel bzw. ihres Einsatzes erachtet wird, untersucht eine Reflexion der Technik als Medium, wie das System der Mittel den Möglichkeitsraum für die Wahl von Mitteln und Zwecken abgibt.“ (Hubig 2006, S. 259). Ihdes machine mediation nimmt im Sinne dieser umfassenden Vermittlung eine Unterposition mit engerem Technikbegriff ein, weshalb er von naked perception sprechen kann. Im Sinne der umfassenderen Position wäre jede vermeintlich nackte Wahrnehmung wiederum eine andere Weise technischer Vermittlung; schließlich weist jeder körpereigene Sensor (Auge, Ohren etc.) ebenfalls spezifische Transformationen auf (Stereohören, zwei Augen mit überlagernden Sichtfeldern, Grenzen spezifischer Frequenzbereiche etc.), die sich technisch erklären lassen. Weiter wäre jedes Handeln - gefasst als intendierte Herbeiführung von Ereignissen - durch die Kategorien von Handlungsmittel und Handlungszweck auf solche technische Vermittlung verwiesen, da Technik mit Hubig allererst den Möglichkeitsraum der Zweck-Mittel-Wahl abgibt.
} 
Zahnoberfläche erspüren kann, die er ohne das Instrument nicht hätte spüren können. Gleichzeitig verschwinden Aspekte aus der Wahrnehmung, die ohne das Instrument durchaus wahrnehmbar wären, wie etwa Temperatur oder Feuchtigkeit des Zahns. In der Wahrnehmung verschwindet jedoch das Instrument und man meint, die Unebenheiten direkt zu spüren. Das Instrument erscheint somit wie ein Teil des eigenen Körpers. Eine zweite Relation bezeichnet Ihde als die hermeneutische Relation (,hermeneutic relation,“ „experience of technical representation of the world"; Ihde 1979, S. 11-13), in der man Welt als durch Technik repräsentiert wahrnimmt und anhand von technischen Informationen (Anzeigen, Monitore etc.) auf die technisch vermittelten Weltzustände schließt. Hierbei muss Technik als Zeichen von Welt interpretiert werden, was diese Relation zu einer hermeneutischen macht. Eine dritte Art der Relation schließlich sieht Ihde in den Hintergrundrelationen (,background relations“; Ihde 1979, S. 13-14), die die vernetzten Systeme der Technosphäre miteinander eingehen, ohne dass der Mensch im Umgang mit ihnen davon erfährt oder sein Handeln explizit auf diese richtet. Bei der Bedienung eines Toasters muss nicht dessen Ermöglichungsstruktur über Stromnetze und Kraftwerke mitberücksichtigt werden. Die Technikentwicklung der letzten Jahrzehnte, vor allem die Entwicklung von hochautomatisierten, vernetzten und lernenden Systemen spricht für eine enorme Ausweitung der Hintergrundrelationen der Technosphäre. Die Alltagsdurchdringung mit Technik - speziell mit lernender Technik - hat nahezu jeden Weltkontakt als Technikkontakt überformt und damit Weltwahrnehmung zu hermeneutischer Technikwahrnehmung transformiert.

Im speziellen Falle lernender Systeme, die vor allem etwas über die Nutzer lernen, zeichnet sich eine Inversion der hermeneutischen Relation ab. In der hermeneutischen Relation repräsentiert Technik Welt und der Mensch interpretiert die Welt über die Technik bzw. im Abgleich verschiedener technischer Repräsentationen von Welt. In der invertierten hermeneutischen Relation repräsentieren Daten Menschen, und die lernende Technik ,interpretiert ${ }^{\text {* }}$ die Menschen über diese Daten. Dabei ist zu berücksichtigen, dass in philosophischem Sinne Interpretieren und Auslegen spezifische Vorbedingungen aufweisen, die nicht ohne weiteres auf Technik übertragen werden können. Daher liegt der These von der invertierten hermeneutischen Relation genau genommen eine Übertragung $\mathrm{zu}$ Grunde, nämlich ein metaphorischer Gebrauch (metapherein bedeutet wörtlich über-tragen) der Aussage Technik interpretiert bzw. legt aus. Da zuvorkommende Technik die Wahrnehmungs-, Entscheidungs- und Handlungsoptionen der Menschen steigend mit dem Grad der cyber-physischen Durchdringung umfassend vorstrukturiert und die datengestützten ,Auslegungen “ der Menschen Teil der technischen Strukturierungsbedingungen sind, begegnen 
Menschen einer Welt, die für sie adaptiert wurde. Die entsprechenden Konzernversprechen besagen, dass diese Adaption eine Personalisierung darstelle und die Menschen so einem individuellen entscheidungsergonomischen Spielraum begegneten. Allerdings sind die Daten, die ein Mensch erzeugt, nicht identisch mit diesem Menschen selbst, sondern nach Digitalisierbarkeit und Nicht-Digitalisierbarkeit, nach Sensorbauart und Kommunikationsstandards etc. selektiert. Auch ist die Auslegung von Daten wiederum nicht identisch mit den Daten selbst, sodass in mehrfacher Hinsicht Diskrepanzen zwischen dem Menschen als Individuum, dem Nutzer als Exemplar (vgl. Gransche und Gethmann 2018, S. 29-36) und der maschinengelernten Weltausrichtung bestehen. Die personenbezogenen, wenn auch stets irgendwie verzerrten Auslegungen sind wiederum nur teilweise Grundlage umfassender Optionsstrukturierung, da auch weitere Profile des Technosphärenkollektivs, z. B. aller Mitglieder einer technischen Lerngemeinschaft (etwa alle Echo-Nutzer, alle Spotify Kunden etc.) sowie zahlende Interessenten (die Nudging-Kunden) diese Strukturierung mitbedingen und diese dabei aber listig dennoch als Ableitung individuellen Agierens präsentiert wird. Die Optionsstrukturierung durch lernende Systeme wird dabei umso wirkungsmächtiger, je weiter die realphysische Anbindung an die Systeme in immer mehr Lebensbereichen voranschreitet. Je mehr unser Weltkontakt primär Technikkontakt wird, desto umfassender werden systemische Vorstrukturierungen von Wahrnehmungs-, Entscheidungs- und Handlungsräumen.

Bei der invertierten hermeneutischen Relation haben wir es daher mit der ,Auslegung' der Person anhand vergangener Präferenzen und Handlungen etc. und anhand ihrer Nutzerprofile als Exemplar zu tun. Dabei kommt vor allem ein Kategorienfehler zum Tragen nämlich, dass qua Datenbasis zwangsläufig immer nur Vorgänge als solche (gefasst als nicht herbeigeführte Ereignisse) ausgelegt werden, denn ob eine Handlung etwa freiwillig, widerwillig, widerstrebend oder gezwungenermaßen ausgeführt wird, ob ein Ereignis überhaupt intendiert war, kann z. B. die entsprechende GPS-Spur nicht erfassen. Von den erfassten Vorgängen werden aber zur Manipulation von Handlungen (intendiert herbeigeführte Ereignisse) Schlüsse gezogen und die Wahrnehmungs-, Entscheidungs- und Handlungsoptionen werden basierend auf diesen Auslegungen (vor-)strukturiert. Daraus resultiert auch für Assistenzrelationen als Fall von Mensch-Technik-Relationen eine normative Beeinflussung bei der Wahl der Handlungszwecke (Zwecksetzungsassistenz) und eine Festlegung der Möglichkeiten der Person auf die daten- und vergangenheitsbasierte Auslegung des Exemplars - und dies unter einkaufbarer Berücksichtigung von listig eingebundenen Drittpräferenzen. 


\section{$5 \quad$ Fazit}

Lernen, besser werden und Kompetenzen bilden war lange Zeit ein Entwicklungspotential, das rein auf der menschlichen Seite von Mensch-TechnikRelationen stattfand. Der Schmied lernte besser hämmern, nicht der Hammer. Mit der Entwicklung und Ausbreitung von lernenden Systemen haben sich auch Entwicklungspotenziale durch die Interaktionssukzession auf Technikseite ergeben. Durch die neuartige Leistungsfähigkeit lernender, autonomer (hochautomatisierter) und vernetzter Technik steigt das Delegierungspotenzial an die Technik, steigt die Assistenzfähigkeit. Aufgaben auf operativer und strategischer Ebene können dabei an Technik delegiert werden, Aufgaben auf normativer Ebene hingegen nicht. Allerdings kann lernende Technik den autonomen Zwecksetzungsprozess unterstützen und somit geleiten und leiten, womit Assistenz sich von der Umsetzungs- und Realisierungsunterstützung auch zur Zwecksetzungsunterstützung ausweitet. Dabei werden Entscheidungsunterstützungen bzw. Empfehlungen als vermeintliche Ableitungen aus den Individuen präsentiert, und die technische Auslegung von Menschen anhand von deren Daten wird (teilweise) zur Grundlage umfassender Weltzurichtung als Assistenzleistung. Vor allem die Verbergensstrategien der Listen, die unlauteren Irreführungen auch durch Verschweigen wichtiger Informationen oder das schwer erkennbare und subtile Nudging machen es äußerst schwierig, das Zusammenwirken von Mensch und Technik bzw. von natürlicher Intelligenz und künstlicher Intelligenz korrekt zu erfassen, entsprechend zu bewerten und im Zweifel zielgerichtet umzugestalten. Es handelt sich bei der Opposition von Mensch und lernenden Systemen weniger, wie oft in der KI-Berichterstattung suggeriert wird, um einen Vorherrschaftskampf beider Intelligenzen; vielmehr stellt sich bei gegenwärtigem Entwicklungsstand lernende Technik v. a. als mächtiges (weil gut verbergendes, listiges) Mittel dar, durch das Menschen andere Menschen beeinflussen können. Damit wäre der Assistenzgedanke - doch etwas erreichen zu können, was man eigentlich nicht erreichen kann, wozu man sich keine Hilfe organisieren oder leisten, sich also weder einigen noch jemand unterdrücken möchte - je nach Position heutiger technischer Macht in zweifacher Weise transformiert: Erstens erreichen mit diesem mächtigen Mittel diejenigen an der technischen Macht ein Entscheiden und Handeln von Anderen nach ihrem Sinne, womit die Systeme für diese Personen zu Machtinstrumenten werden, was sich nicht zuletzt deutlich in bislang beispiellosen Einfluss- und Reichtumskonzentrationen zeigt. Zweitens erreichen alle anderen, der überlistete Treibstoff gewissermaßen, zwar, was der Kerngedanke ausdrückte: Erreichen, was man will, mehr als man kann und 
sich dabei nicht einigen, auf Kompromisse einlassen oder andere unterdrücken müssen. Nur hat sich über die zuvorkommende Technik eine Manipulierbarkeit der Entscheidungen im Bereich der Zwecksetzungsautonomie eingestellt, sodass man zwar alleine - lediglich mit technischer Unterstützung - erreichen kann, was man will, man aber nicht mehr alleine wollen kann, was man will.

\section{Literatur}

Abbott, D. (2014). Applied predictive analytics. Principles and techniques for the professional data analyst. Indianapolis: Wiley.

acatech (Hrsg.). (2015). Neue autoMobilität. Automatisierter Straßenverkehr der Zukunft. Unter Mitarbeit von Michael Püschner, Stefanie Baumann und Tobias Hesse. Deutsche Akademie der Technikwissenschaften. München: Utz (acatech Position). Zugegriffen: 3. März 2018.

Bundesamt für Justiz. (2004a). Gesetz gegen den unlauteren Wettbewerb § 5 a UWG Irreführung durch Unterlassen. https://www.gesetze-im-internet.de/uwg_2004/_5a.html. Zugegriffen: 15. Apr. 2019.

Bundesamt für Justiz. (2004b). Gesetz gegen den unlauteren Wettbewerb: § 5 UWG Irreführende geschäftliche Handlungen. https://www.gesetze-im-internet.de/uwg_2004/_5. html. Zuletzt aktualisiert und geprüft am 15. Apr. 2019.

Bundesregierung. (2018). Strategie Künstliche Intelligenz der Bundesregierung. https:// www.bmbf.de/files/Nationale_KI-Strategie.pdf. Zugegriffen: 12. Juli 2019.

Cachada, A., Barbosa, J., Leitno, P., Gcraldes, C. A. S., Deusdado, L., Costa, J. et al. (2018). Maintenance 4.0: Intelligent and predictive maintenance system architecture. In 2018 IEEE 23rd International Conference on Emerging Technologies and Factory Automation (ETFA). Proceedings : Politecnico di Torino, Torino, Italy, 04-07 September, 2018. 2018 IEEE 23rd International Conference on Emerging Technologies and Factory Automation (ETFA). Turin, 9/4/2018-9/7/2018 (S. 139-146). Piscataway: IEEE.

Davis, S. M. (1997). Future perfect. Reading: Addison.

Duportail, J. (2017). I asked Tinder for my data. It sent me 800 pages of my deepest, darkest secrets. The guardian. https://www.theguardian.com/technology/2017/sep/26/ tinder-personal-data-dating-app-messages-hacked-sold. Zugegriffen: 12. Juli 2019.

Endsely, M. R., \& Kaber, D. B. (1999). Level of automation effects on performance, situation awareness and workload in a dynamic control task. Ergonomics, 42(3), 462-492.

Gottschalk-Mazouz, N. (2008). „Autonomie“ und die Autonomie „autonomer technischer Systeme“. XXI. Deutscher Kongress für Philosophie: Lebenswelt und Wissenschaft. DGPhil2008. https://www.dgphil2008.de/fileadmin/download/ Sektionsbeitraege/07_Gottschalk-Mazouz.pdf. Zuletzt aktualisiert am 30. Juli 2008, Zuletzt geprüft am 13. Juli 2019.

Gransche, B. (2020). Technogene Unheimlichkeit. In A. Friedrich, P. Gehring, C. Hubig, A. Kaminski, \& A. Nordmann (Hrsg.), Unheimlichkeit und Autonomie. Jahrbuch Technikphilosophie 2020. Baden-Baden: Nomos, 33-51. 
Gransche, B., \& Gethmann, C. F. (2018). Digitalisate zwischen Erklären und Verstehen. Chancen und Herausforderungen durch Big Data für die Kultur- und Sozialwissenschaften - Eine wissenschaftstheoretische Desillusionierung. https://www.abida.de/ sites/default/files/ABIDA\%20Gutachten\%20Digitalisate.pdf. Zuletzt aktualisiert am 31. März 2018, Zuletzt geprüft am 28. Febr. 2019.

Gransche, B., Shala, E., Hubig, C., et al. (2014). Wandel von Autonomie und Kontrolle durch пеие Mensch-Technik-Interaktionen. Grundsatzfragen autonomieorientierter Mensch-Technik-Verhältnisse. Stuttgart: Fraunhofer.

Hegel, G. W. F. (1999). Wissenschaft der Logik II. Erster Teil. Die objektive Logik. Zweites Buch. Zweiter Teil. Die subjektive Logik (2.-5. Aufl.). Frankfurt a. M.: Suhrkamp.

Hightech Forum \& acatech. (2017). Fachforum Autonome Systeme. Chancen und Risiken für Wirtschaft, Wissenschaft und Gesellschaft. Abschlussbericht - Langversion. Zugegriffen: 12. Juli 2019.

Hubig, C. (2006). Die Kunst des Möglichen I. Grundlinien einer dialektischen Philosophie der Technik; Technikphilosophie als Reflexion der Medialität (Bd. 2). Bielefeld: transcript.

Hubig, C. (2011). „Natur“ und Kultur. Von Inbegriffen zu Reflexionsbegriffen. ZKphil, 5(1), 97-119.

Husserl, E. G. (1891). Philosophie der Arithmetik. Psychologische und logische Untersuchungen. Halle (Saale): Pfeiffer (1). urn:nbn:de:bsz:25-opus-61596. Zugegriffen: 13. Jan. 2013.

Ihde, D. (1979). Technics and praxis. Dordrecht: Reidel (Synthese library, 130).

Kaber, D., \& Prinzel, L. J. (2006). Adaptive and adaptable automation design. A critical review of the literature and recommendations for future research (NASA/TM-2006214504). Zugegriffen: 10. Juli 2019.

Kant, I. (1798). Der Streit der Fakultäten, Anthropologie in pragmatischer Hinsicht. Immanuel Kant: Gesammelte Werke. Akademieausgabe - Elektronische Edition, VII. https://korpora.zim.uni-duisburg-essen.de/kant/aa07/Inhalt7.html.

Kirchner, F., Michaelis, K. T., Hoffmeister, J., Regenbogen, A., \& Meyer, U. (Hrsg.). (1998). Wörterbuch der philosophischen Begriffe. Bd. 500: Philosophische Bibliothek. Hamburg: Meiner.

Mähler, R., \& Vonderau, P. (2017). Studying ad targeting with digital methods: The case of Spotifyi. Culture Unbound 9(2), 212-221. Zugegriffen: 10. Dez. 2018.

Münzer, S., Zimmer, H. D., \& Baus, J. (2012). Navigation assistance: A trade-off between wayfinding support and configural learning support. Journal of Experimental Psychology. Applied, 18(1), 18-37. https://doi.org/10.1037/a0026553

OECD. (2019). Empfehlung des Rats zu künstlicher Intelligenz. https://www.oecd.org/ berlin/presse/Empfehlung-des-Rats-zu-kuenstlicher-Intelligenz.pdf. Zugegriffen: 12. Juli 2019.

Peppers, D., \& Rogers, M. (1997). The one to one future. Building relationships one customer at a time (1. Aufl.). New York: Currency Doubleday.

Pfaller, R. (2018). Erwachsenensprache. Über ihr Verschwinden aus Politik und Kultur (3. Aufl.). Frankfurt a. M.: Fischer. 
Schilling, M., Kopp, S., Wachsmuth, S., Wrede, B., Ritter, H., Brox, T., et al. (2016). Towards a multidimensional perspective on shared autonomy. Technical report FS-16-05. The 2016 AAAI fall symposium series: Shared autonomy in research and practice, S. 338-344.

Sheridan, T. B., \& Verplank, W. L. (1978). Human and computer control of undersea teleoperators. MIT Man-Machine Systems Lab. Cambridge: Massachusetts Institute of Technology. Zugegriffen: 12. Juli 2019.

spotify. (2018). Spotify for Brands. Zielgruppen. https://spotifyforbrands.com/de-DE/ audiences/. Zuletzt aktualisiert am 8. Mai 2018, Zuletzt geprüft am 14. Mai 2018.

spotify. (2019). Musik für alle. https://www.spotify.com/de/premium/?checkout=false. Zugegriffen: 15. Apr. 2019.

spotify for brands. (2018). Audiences. https://spotifyforbrands.com/en-GB/audiences. Zugegriffen: 3. Dez. 2018.

von Goethe, J. W. (1986). Faust. Der Tragödie erster Teil (Universal-Bibliothek, Bd. 1, Durchges. Ausg). Stuttgart: Reclam.

Wadivkar, O. (2019). Predictive pricing hits bigtime in chemicals. https://www.accenture. com/us-en/blogs/blogs-predictive-pricing-hits-bigtime-chemicals. Zugegriffen: 12. Juli 2019.

Yan, J., Meng, Y., Lu, L., \& Li, L. (2017). Industrial big data in an industry 4.0 environment. Challenges, schemes, and applications for predictive maintenance. IEEE Access, 5, 23484-23491. https://doi.org/10.1109/ACCESS.2017.2765544

Open Access Dieses Kapitel wird unter der Creative Commons Namensnennung 4.0 International Lizenz (http://creativecommons.org/licenses/by/4.0/deed.de) veröffentlicht, welche die Nutzung, Vervielfältigung, Bearbeitung, Verbreitung und Wiedergabe in jeglichem Medium und Format erlaubt, sofern Sie den/die ursprünglichen Autor(en) und die Quelle ordnungsgemäß nennen, einen Link zur Creative Commons Lizenz beifügen und angeben, ob Änderungen vorgenommen wurden.

Die in diesem Kapitel enthaltenen Bilder und sonstiges Drittmaterial unterliegen ebenfalls der genannten Creative Commons Lizenz, sofern sich aus der Abbildungslegende nichts anderes ergibt. Sofern das betreffende Material nicht unter der genannten Creative Commons Lizenz steht und die betreffende Handlung nicht nach gesetzlichen Vorschriften erlaubt ist, ist für die oben aufgeführten Weiterverwendungen des Materials die Einwilligung des jeweiligen Rechteinhabers einzuholen.

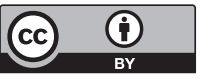

